\title{
Hankel operators on Fock spaces
}

\author{
Article
}

Accepted Version

Perala, A., Schuster, A. and Virtanen, J. A. (2014) Hankel operators on Fock spaces. Operator Theory: Advances and Applications, 236. pp. 377-390. ISSN 0255-0156 doi: https://doi.org/10.1007/978-3-0348-0648-0_24 Available at https://centaur.reading.ac.uk/29217/

It is advisable to refer to the publisher's version if you intend to cite from the work. See Guidance on citing.

To link to this article DOI: http://dx.doi.org/10.1007/978-3-0348-0648-0_24

Publisher: Springer Basel

All outputs in CentAUR are protected by Intellectual Property Rights law, including copyright law. Copyright and IPR is retained by the creators or other copyright holders. Terms and conditions for use of this material are defined in the End User Agreement.

\section{www.reading.ac.uk/centaur}

\section{CentAUR}

Central Archive at the University of Reading

Reading's research outputs online 


\title{
Hankel operators on Fock spaces
}

\author{
A. Perälä, A. Schuster and J. A. Virtanen
}

\begin{abstract}
We study Hankel operators on the weighted Fock spaces $F_{\gamma}^{p}$. The boundedness and compactness of these operators are characterized in terms of $B M O$ and $V M O$, respectively. Along the way, we also study Berezin transform and harmonic conjugates on the plane. Our results are analogous to Zhu's characterization of bounded and compact Hankel operators on Bergman spaces of the unit disk.
\end{abstract}

Mathematics Subject Classification (2000). Primary 47B35; Secondary 30H20, $30 \mathrm{H} 35$.

Keywords. Hankel operators, Fock spaces, boundedness, compactness.

\section{Introduction}

Hankel operators have been studied for several decades in the setting of various analytic function spaces. Starting with Hankel matrices, which can be viewed as Hankel operators on Hardy spaces (see [9]), the field has expanded to Hankel operators on Bergman spaces, Dirichlet type spaces, Bergman and Hardy spaces of the unit ball in $\mathbb{C}^{n}$, of symmetric domains, and Fock spaces. In addition to being a beautiful and rapidly developing part of analysis, Hankel operators have a vast number of applications, which in the case of Hardy spaces are well known and recognized (see, e.g. [9]), while Hankel operators on Bergman and Fock spaces have found applications mainly in quantum mechanics.

We are interested in the basic properties of Hankel operators on Fock spaces, and in particular characterize their boundedness and compactness in terms of the (mean) oscillation of the generating symbols. In the Bergman space setting one is led to the space of bounded mean oscillation $B M O_{\partial}^{p}$ and the space of vanishing mean oscillation $V M O_{\partial}^{p}$ with respect to the Bergman metric. Due to K. Zhu [12], a characterization of bounded and compact Hankel operators has been known for

The first author acknowledges support by the Academy of Finland project no. 75166001. The third author was supported by a Marie Curie International Outgoing Fellowship within the 7th European Community Framework Programme. 
two decades. It is natural to ask whether an analogous result carries over to Fock spaces. Indeed, the question was recently settled in [2] for the Hilbert Fock space $F^{2}$.

For a symbol $f$ (satisfying suitable conditions), we define the Hankel operator $H_{f}$ by

$$
H_{f}=(I-P) M_{f}
$$

where $P$ is a projection defined below in (1) and $M_{f}$ is the operator of multiplication associated with $f$. In this paper we study Hankel operators on standard Fock spaces $F_{\gamma}^{p}$ with $1 \leq p<\infty$ and $\gamma>0$. We will introduce spaces $B M O^{p}$ and $V M O^{p}$ (in the Euclidean metric) and obtain useful characterizations for these spaces. We prove decomposition theorems similar to those in $[11,12]$; in particular, we show that these spaces can be characterized in terms of certain Gaussian integrals, where $\gamma>0$ can be arbitrary.

Note that the John-Nirenberg theorem implies that the classical BMO and $V M O$ spaces are independent of the parameter $p$. However, as in the case of the Bergman metric, the spaces $B M O^{p}$ and $V M O^{p}$ presented here depend on $p$.

\section{The weighted Fock spaces}

We will use the definitions from [7]. Let $\gamma>0$ and $1 \leq p<\infty$. The weighted Fock space $F_{\gamma}^{p}$ consists of entire functions $f$ such that

$$
\|f\|_{p, \gamma}^{p}=\int_{\mathbb{C}}|f(z)|^{p} e^{-(\gamma p / 2)|z|^{2}} d A(z)<\infty
$$

Here $d A(z)=d x d y$ is the standard Lebesgue area measure. Similarly, the space $F_{\gamma}^{\infty}$ consists of those entire $f$, for which

$$
\|f\|_{\infty, \gamma}=\sup _{z \in \mathbb{C}}|f(z)| e^{-(\gamma / 2)|z|^{2}}
$$

is finite. The respective Lebesgue $L_{\gamma}^{p}$ spaces and their norms are defined in an obvious way.

It is known that $F_{\gamma}^{2}$ is a Hilbert space with inner product

$$
\langle f, g\rangle=\int_{\mathbb{C}} f(z) \overline{g(z)} e^{-\gamma|z|^{2}} d A(z) .
$$

REMARK. The point-evaluation functionals $f \mapsto f(z)$ are bounded $F_{\gamma}^{p} \rightarrow \mathbb{C}$ are bounded and hence $F_{\gamma}^{2}$ is known to possess reproducing kernels $K_{z}:=K_{z}^{\gamma} ; f(z)=$ $\left\langle f, K_{z}\right\rangle$.

One immediate corollary is that norm convergence implies locally uniform convergence. In other words, if $f_{n}$ and $f$ are in $F_{\gamma}^{p}$ and $\left\|f_{n}-f\right\|_{p, \gamma} \rightarrow 0$ as $n \rightarrow \infty$, then $f_{n}(z) \rightarrow f(z)$ uniformly on each compact subset of $\mathbb{C}$. Another corollary is that the space $F_{\gamma}^{p}$ is complete; if $\left\{f_{n}\right\}$ is a Cauchy sequence in norm, then $f_{n} \rightarrow f$ in norm for some $f \in F_{\gamma}^{p}$. 
The reproducing kernels $K_{z}$ can be explicitly computed; $K_{z}(w)=e^{\gamma \bar{z} w}$. The Bergman projection $P:=P_{\gamma}$ is given by

$$
P f(z)=\int_{\mathbb{C}} f(w) e^{\gamma z \bar{w}} e^{-\gamma|w|^{2}} d A(w) .
$$

It is known that $P: L_{\gamma}^{p} \rightarrow F_{\gamma}^{p}$ is bounded for every $\gamma>0$ and $p \in[1, \infty]$. Proofs can be found in [5]. We will just write $K_{z}$ and $P$, instead of $K_{z}^{\gamma}$ and $P_{\gamma}$; the parameter $\gamma$ will be clear from context.

A measurable function $f$ is said to belong to $\tau^{p}=\tau_{\gamma}^{p}$ if and only if $f K_{z} \in L_{\gamma}^{p}$ for every $z \in \mathbb{C}$. This requirement is natural, since linear combinations of the kernel functions form a dense subset of $F_{\gamma}^{p}$. Henceforth, we will usually assume $f \in \tau^{p}$.

\section{BMO and related spaces}

For $0<r<\infty$, let $D(z, r)$ be the Euclidean disk of radius $r$ and center $z$. For $f \in L_{\text {loc }}^{1}, 0<r<\infty, z \in \mathbb{C}$, let

$$
\widehat{f}_{r}(z)=\frac{1}{\pi r^{2}} \int_{D(z, r)} f(w) d A(w)
$$

Fix $0<r<\infty$ and $p \geq 1$. Define $B M O_{r}^{p}$ to be the set of $L_{l o c}^{p}$ integrable functions $f$ such that

$$
\|f\|_{B M O_{r}^{p}}=\sup _{z \in \mathbb{C}}\left\{\frac{1}{\pi r^{2}} \int_{D(z, r)}\left|f(w)-\widehat{f}_{r}(z)\right|^{p} d A(w)\right\}^{\frac{1}{p}}<\infty .
$$

Let $\mathrm{BO}_{r}$ be the set of continuous functions in $\mathbb{C}$ such that

$$
\|f\|_{B O_{r}}=\sup _{z \in \mathbb{C}} \omega_{r}(f)<\infty
$$

where

$$
\omega_{r}(f)(z)=\sup _{w \in D(z, r)}|f(z)-f(w)|
$$

Let $B A_{r}^{p}$ be the set of functions $f$ on $\mathbb{C}$ such that $\widehat{f}_{r}$ is bounded on $\mathbb{C}$.

Lemma 3.1. Let $f \in L_{l o c}^{p}$. Then $f \in B M O_{r}^{p}$ if and only if there is a constant $C>0$ such that for every $z \in \mathbb{C}$ there is a constant $\lambda_{z}$ such that

$$
\frac{1}{\pi r^{2}} \int_{D(z, r)}\left|f(w)-\lambda_{z}\right|^{p} d A(w) \leq C
$$


Proof. For the proof of the forward direction, let $\lambda_{z}=\widehat{f}_{r}(z)$. For the other direction, note that

$$
\begin{aligned}
& \left\{\frac{1}{\pi r^{2}} \int_{D(z, r)}\left|f(w)-\widehat{f}_{r}(z)\right|^{p} d A(w)\right\}^{\frac{1}{p}} \\
\leq & \left\{\frac{1}{\pi r^{2}} \int_{D(z, r)}\left|f(w)-\lambda_{z}\right|^{p} d A(w)\right\}^{\frac{1}{p}} \\
+ & \left\{\frac{1}{\pi r^{2}} \int_{D(z, r)}\left|\lambda_{z}-\widehat{f}_{r}(z)\right|^{p} d A(w)\right\}^{\frac{1}{p}} \\
= & \left\{\frac{1}{\pi r^{2}} \int_{D(z, r)}\left|f(w)-\lambda_{z}\right|^{p} d A(w)\right\}^{\frac{1}{p}}+\left|\lambda_{z}-\widehat{f}_{r}(z)\right| .
\end{aligned}
$$

But

$$
\begin{aligned}
\left|\lambda_{z}-\widehat{f}_{r}(z)\right| & =\left|\frac{1}{\pi r^{2}} \int_{D(z, r)}\left(f(w)-\lambda_{z}\right) d A(w)\right| \\
& \leq\left\{\frac{1}{\pi r^{2}} \int_{D(z, r)}\left|\lambda_{z}-f(w)\right|^{p} d A(w)\right\}^{\frac{1}{p}}
\end{aligned}
$$

Therefore

$$
\left\{\frac{1}{\pi r^{2}} \int_{D(z, r)}\left|f(w)-\widehat{f}_{r}(z)\right|^{p} d A(w)\right\}^{\frac{1}{p}} \leq 2\left\{\frac{1}{\pi r^{2}} \int_{D(z, r)}\left|\lambda_{z}-f(w)\right|^{p} d A(w)\right\}^{\frac{1}{p}} .
$$

Lemma 3.2. Let $s>r>0$. Then $B M O_{s}^{p} \subset B M O_{r}^{p}$.

Proof. Suppose $f \in B M O_{s}^{p}$ so that for every $z \in \mathbb{C}$ we have $\lambda_{z} \in \mathbb{C}$ such that

$$
\sup _{z \in \mathbb{C}} \frac{1}{\pi s^{2}} \int_{D(z, s)}\left|f(w)-\lambda_{z}\right|^{p} d A(w)=C<\infty .
$$

Now

$$
\begin{aligned}
& \frac{1}{\pi r^{2}} \int_{D(z, r)}\left|f(w)-\lambda_{z}\right|^{p} d A(w) \\
\leq & \frac{s^{2}}{r^{2}} \frac{1}{\pi s^{2}} \int_{D(z, s)}\left|f(w)-\lambda_{z}\right|^{p} d A(w) \\
\leq & C \frac{s^{2}}{r^{2}}
\end{aligned}
$$

for every $z \in \mathbb{C}$.

Lemma 3.3. $B O_{r}$ is independent of $r$. 
Proof. Let $r<s$. Then $\|f\|_{B O_{r}} \leq\|f\|_{B O_{s}}$.

Choose $N \in \mathbb{N}$ such that for any $w \in D(0, s)$, there exists a path $\{0=$ $\left.z_{1}, z_{2}, \ldots, z_{N}=w\right\}$ in $D(0, s)$ such that $\left|z_{i-1}-z_{i}\right|<r$. Let now $z \in \mathbb{C}$. Then for any $w \in D(z, s)$, we have a path $\left\{z=\zeta_{1}, \zeta_{2}, \ldots, \zeta_{N}=w\right\}$, where $\zeta_{i}=z_{i}+z$, and $\left|\zeta_{i-1}-\zeta_{i}\right|<r$. Therefore

$$
|f(z)-f(w)| \leq \sum_{i=1}^{N}\left|f\left(\zeta_{i-1}\right)-f\left(\zeta_{i}\right)\right| \leq N \sup \left\{w_{r}(f)\left(\zeta_{i}\right): i\right\} \leq N\|f\|_{B O_{r}} .
$$

We now take the supremum over all $w \in D(z, r)$ and then over all $z \in \mathbb{C}$ to obtain the desired result.

By the above lemma, we shall now refer to $B O=B O_{1}$.

Lemma 3.4. Let $f$ be a continuous function on $\mathbb{C}$. Then $f \in B O$ if and only if there is a constant $C>0$ such that

$$
|f(z)-f(w)| \leq C(|z-w|+1)
$$

for all $z, w \in \mathbb{C}$.

Proof. The backward direction is obviously true. For the forward direction, let $w, z \in \mathbb{C}$. If $f \in B O$, then

$$
C \geq \sup _{\alpha \in \mathbb{C}} \omega_{1}(f)(\alpha)=\sup _{\alpha \in \mathbb{C}} \sup _{w \in D(\alpha, \beta)<1}|f(\alpha)-f(\beta)| .
$$

In other words, if $|z-w| \leq 1$, then $|f(z)-f(w)| \leq C \leq C(|z-w|+1)$. Suppose now that $|z-w|>1$. Let $N=[|z-w|]+1$, where $[x]$ is the greatest integer less than or equal to $x$. Let $z_{0}=z, z_{1}$ be the point a distance of $|z-w| / N$ along the line from $z$ to $w$. Let $z_{2}$ be the point a distance of $|z-w| / N$ along the line from $z_{1}$ to $w$, and so on, until $z_{N}=w$. Then

$$
|f(z)-f(w)| \leq \sum_{i=1}^{N}\left|f\left(z_{i-1}\right)-f\left(z_{i}\right)\right| \leq N\|f\|_{B O} \leq\|f\|_{B O}(|z-w|+1) .
$$

Let $B A_{r}^{p}$ denote the space of all functions $f$ on $\mathbb{C}$ such that

$$
\|f\|_{B A_{r}^{p}}=\sup _{z \in \mathbb{C}}\left\{{\widehat{|f|^{p}}}_{r}(z)\right\}^{\frac{1}{p}}<\infty .
$$

In other words, $f \in B A_{r}^{p}$ if

$$
\frac{1}{\pi r^{2}} \int_{D(z, r)}|f(w)|^{p} d A(w)
$$

is bounded independently of $z \in \mathbb{C}$. The notion of $B A_{r}^{p}$ is closely related to Carleson measures on Fock spaces, see [7], or [10] for more generality.

Lemma 3.5. Let $r>0$. Then $f \in B A_{r}^{p}$ if and only if $M_{f}: F_{\gamma}^{p} \rightarrow L_{\gamma}^{p}$ is bounded. 
Proof. Let $d \mu(w)=|f(w)|^{p} d A(w)$. Then

$$
\left\{\widehat{|f|}_{r}^{p}(z)\right\}=\frac{1}{\pi r^{2}} \int_{D(z, r)}|f(w)|^{p} d A(w)
$$

Then

$$
\mu(D(z, r))=\int_{D(z, r)} d \mu(w)=\int_{D(z, r)}|f(w)|^{p} d A(w) .
$$

Of course this implies that $\mu$ is a Carleson measure if and only if $f \in B A_{r}^{p}$. But this means

$$
\begin{aligned}
& \int_{\mathbb{C}}|g(w)|^{p} e^{-(\gamma p / 2)|w|^{2}}|f(w)|^{p} d A(w) \\
= & \int_{\mathbb{C}}|g(w)|^{p} e^{-(\gamma p / 2)|w|^{2}} d \mu(w) \\
\leq & C \int_{\mathbb{C}}|g(w)|^{p} e^{-(\gamma p / 2)|w|^{2}} d A(w)
\end{aligned}
$$

for all $g \in F_{\gamma}^{p}$. In other words, $M_{f}: F_{\gamma}^{p} \rightarrow L_{\gamma}^{p}$ is bounded.

LEMMA 3.6. If $f \in B M O_{2 r}^{p}$, then $\widehat{f}_{r} \in B O_{r}$.

Proof. Let $f \in B M O_{2 r}^{p}$, and suppose $|w-z| \leq r$. Then

$$
\begin{aligned}
\left|\widehat{f}_{r}(z)-\widehat{f}_{r}(w)\right| & \leq\left|\widehat{f}_{r}(z)-\widehat{f}_{2 r}(z)\right|+\left|\widehat{f}_{2 r}(z)-\widehat{f}_{r}(w)\right| \\
& =\left|\frac{1}{\pi r^{2}} \int_{D(z, r)} f(u) d A(u)-\frac{1}{\pi r^{2}} \int_{D(z, r)} \widehat{f}_{2 r}(z) d A(u)\right| \\
& +\left|\frac{1}{\pi r^{2}} \int_{D(w, r)} f(u) d A(u)-\frac{1}{\pi r^{2}} \int_{D(w, r)} \widehat{f}_{2 r}(z) d A(u)\right| \\
& \leq \frac{1}{\pi r^{2}} \int_{D(z, r)}\left|f(u)-\widehat{f}_{2 r}(z)\right| d A(u) \\
& +\frac{1}{\pi r^{2}} \int_{D(w, r)}\left|f(u)-\widehat{f}_{2 r}(z)\right| d A(u) \\
& \leq 4 \frac{1}{4 \pi r^{2}} \int_{D(z, 2 r)}\left|f(u)-\widehat{f}_{2 r}(z)\right| d A(u) \\
& +4 \frac{1}{4 \pi r^{2}} \int_{D(z, 2 r)}\left|f(u)-\widehat{f}_{2 r}(z)\right| d A(u) \\
& \leq 4\left\{\frac{1}{\pi 4 r^{2}} \int_{D(z, 2 r)}\left|f(u)-\widehat{f}_{2 r}(z)\right|^{p} d A(u)\right\}^{\frac{1}{p}} \\
& +4\left\{\frac{1}{\pi 4 r^{2}} \int_{D(z, 2 r)}\left|f(u)-\widehat{f}_{2 r}(z)\right|^{p} d A(u)\right\}^{\frac{1}{p}} \\
& \leq 8\|f\|_{B M O_{2 r}^{p} .}
\end{aligned}
$$


The fourth line follows from the fact that $D(z, r) \subset D(z, 2 r)$ and $D(w, r) \subset D(z, r)$ and the fifth follows from Hölder's inequality.

Let $k_{z}=K_{z} /\left\|K_{z}\right\|_{2, \gamma}$, so that $k_{z}(w)=e^{\gamma \bar{z} w-(\gamma / 2)|z|^{2}}$ denote the normalized reproducing kernel of $F_{\gamma}^{2}$. An easy calculation reveals that $k_{z}=k_{z}^{\gamma}$ is a unit vector on $F_{\gamma}^{p}$ for every $p \in[1, \infty)$.

The Berezin transform (or the heat-transform) of a function $f$ is given by

$$
B_{\gamma} f(z)=\int_{\mathbb{C}} f(w)\left|k_{z}^{\gamma}(w)\right|^{2} e^{-\gamma|w|^{2}} d A(w) .
$$

We will omit the $\gamma$, when it is clear form the context. In this case we just write $B f$.

Lemma 3.7. Let $f \in \tau^{p}$. Then the following are equivalent.

(1) $f \in B A_{r}^{p}$;

(2) $\sup _{z \in \mathbb{C}} \int_{\mathbb{C}}|f(z-w)|^{p} e^{-\gamma|w|^{2}} d A(w) \leq C$ for some $\gamma>0$;

(3) $\sup _{z \in \mathbb{C}} \int_{\mathbb{C}}|f(z-w)|^{p} e^{-\gamma|w|^{2}} d A(w) \leq C$ for all $\gamma>0$.

Proof. By the definition of $B A_{r}^{p}, f \in B A_{r}^{p}$ if and only if $\int_{D(z, r)}|f(w)|^{p} d A(w) \leq C$ if and only if $|f|^{p} d A$ is a Carleson measure for $F_{\gamma}^{2}$ for some (and thus for every) $\gamma>0$ if and only if the Berezin transform $B_{\gamma}|f|^{p}$ is bounded. But

$$
\begin{aligned}
B_{\gamma}|f|^{p}(z) & =\int_{\mathbb{C}}\left|k_{z}(w)\right|^{2} e^{-\gamma|w|^{2}}|f(w)|^{p} d A(w) \\
& =\int_{\mathbb{C}} e^{-\gamma|z|^{2}+2 \gamma \Re \bar{z} w-\gamma|w|^{2}}|f(w)|^{p} d A(w) \\
& =\int_{\mathbb{C}} e^{-\gamma|z-w|^{2}}|f(w)|^{p} d A(w) \\
& =\int_{\mathbb{C}} e^{-\gamma|w|^{2}}|f(z-w)|^{p} d A(w) .
\end{aligned}
$$

Note that by Lemmas 3.3 and 3.5, both $B O_{r}$ and $B A_{r}^{p}$ are independent of $r$. In fact, if we combine Lemmas 3.5 and 3.7 , we obtain the following lemma.

Lemma 3.8. Let $f \in \tau^{p}$. The following conditions are equivalent:

(1) $f \in B A_{r}^{p}$;

(2) $\sup _{z \in \mathbb{C}} \int_{\mathbb{C}}|f(z-w)|^{p} e^{-\gamma|w|^{2}} d A(w)<\infty$ for some (and thus all) $\gamma>0$;

(3) $M_{f}: F_{\gamma}^{p} \rightarrow L_{\gamma}^{p}$ is bounded.

LEMMA 3.9. If $f \in B M O_{2 r}^{p}$, then $f-\widehat{f}_{r} \in B A^{p}$. 
Proof. By assumption and Lemma 3.2, $f \in B M O_{r}^{p}$. Let $g=f-\widehat{f}_{r}$. Then

$$
\begin{aligned}
& \left\{\widehat{|g|^{p}}{ }_{r}(z)\right\}^{\frac{1}{p}}=\left\{\frac{1}{\pi r^{2}} \int_{D(z, r)}\left|f(u)-\widehat{f}_{r}(u)\right|^{p} d A(u)\right\}^{\frac{1}{p}} \\
& \leq\left\{\frac{1}{\pi r^{2}} \int_{D(z, r)}\left|f(u)-\widehat{f}_{r}(z)\right|^{p} d A(u)\right\}^{\frac{1}{p}} \\
& +\left\{\frac{1}{\pi r^{2}} \int_{D(z, r)}\left|\widehat{f}_{r}(z)-\widehat{f}_{r}(u)\right|^{p} d A(u)\right\}^{\frac{1}{p}} \\
& \leq\|f\|_{B M O_{r}^{p}}+\omega_{r}\left(\widehat{f_{r}}\right)(z) .
\end{aligned}
$$

Lemma 3.10. Let $r>0$. Then

$$
B M O_{r}^{p} \subset B O_{r}+B A_{r}^{p} .
$$

Proof. Let $r=2 s$ and $f \in B M O_{r}^{p}=B M O_{2 s}^{p}$. Then Lemmas 3.6 and 3.9 imply that $\widehat{f}_{s} \in B O_{s}$ and $f-\widehat{f}_{s} \in B A_{s}^{p}$. Therefore, $f=\widehat{f}_{s}+f-\widehat{f}_{s} \in B O_{s}+B A_{s}^{p}=$ $B O_{r}+B A_{r}^{p}$.

LEMMA 3.11. If $f \in B M O_{r}^{p}$, then

$$
\int_{\mathbb{C}}\left|f(z-w)-B_{\gamma} f(z)\right|^{p} e^{-\gamma|w|^{2}} d A(w) \leq C
$$

for all $z \in \mathbb{C}$ and $\gamma>0$.

Proof. By Lemma 3.10, it is enough to show the inequality holds for $f \in B A^{p}$ and $f \in B O$. Suppose first that $f \in B A^{p}$. By Hölder,

$$
\left|B_{\gamma} f(z)\right| \leq C\left\{\int_{\mathbb{C}}|f(z-w)|^{p} e^{-\gamma|w|^{2}} d A(w)\right\}^{\frac{1}{p}} .
$$

Therefore

$$
\begin{aligned}
& \left\{\int_{\mathbb{C}}\left|f(z-w)-B_{\gamma} f(z)\right|^{p} e^{-\gamma|w|^{2}} d A(w)\right\}^{\frac{1}{p}} \\
\leq & \left\{\int_{\mathbb{C}}|f(z-w)|^{p} e^{-\gamma|w|^{2}} d A(w)\right\}^{\frac{1}{p}}+\left|B_{\gamma} f(z)\right| \\
\leq & \left(1+C^{\prime}\right)\left\{\int_{\mathbb{C}}|f(z-w)|^{p} e^{-\gamma|w|^{2}} d A(w)\right\}^{\frac{1}{p}} \leq C,
\end{aligned}
$$

where the last inequality follows from Lemma 3.7 . 
Suppose next that $f \in B O$. Then

$$
\begin{aligned}
& \int_{\mathbb{C}}\left|f(z-w)-B_{\gamma} f(z)\right|^{p} e^{-\gamma|w|^{2}} d A(w) \\
= & \int_{\mathbb{C}}\left|f(z-w)-\int_{\mathbb{C}} f(z-u) e^{-\gamma|u|^{2}} d A(u)\right|^{p} e^{-\gamma|w|^{2}} d A(w) \\
\leq & C \int_{\mathbb{C}} \int_{\mathbb{C}}|f(z-w)-f(z-u)|^{p} e^{-\gamma|w|^{2}} d A(w) e^{-\gamma|u|^{2}} d A(u) .
\end{aligned}
$$

Since $f \in B O$, Lemma 3.4 tells us that $|f(z-w)-f(z-u)|^{p} \leq C(|w-u|+1)^{p}$. Therefore, the last quantity in the last displayed equation is bounded above by

$$
C^{2} \int_{\mathbb{C}} \int_{\mathbb{C}}(|u-w|+1)^{p} e^{-\gamma|w|^{2}} d A(w) e^{-\gamma|u|^{2}} d A(u),
$$

which is a constant.

Lemma 3.12. Suppose there exists $\gamma>0$ such that

$$
\int_{\mathbb{C}}\left|f(z-w)-B_{\gamma} f(z)\right|^{p} e^{-\gamma|w|^{2}} d A(w) \leq C
$$

for all $z \in \mathbb{C}$. Then $f \in B M O_{r}^{p}$.

Proof. Let $z \in \mathbb{C}$ and fix $\gamma>0$. Note that $e^{-\gamma|z-w|^{2}} \geq c>0$ for $w \in D(z, r)$. Therefore

$$
\begin{aligned}
& c \int_{D(z, r)}\left|f(w)-B_{\gamma} f(z)\right|^{p} d A(w) \\
\leq & \int_{D(z, r)}\left|f(w)-B_{\gamma} f(z)\right|^{p} e^{-\gamma|z-w|^{2}} d A(w) \\
\leq & \int_{\mathbb{C}}\left|f(w)-B_{\gamma} f(z)\right|^{p} e^{-\gamma|z-w|^{2}} d A(w) \\
= & \int_{\mathbb{C}}\left|f(z-w)-B_{\gamma} f(z)\right|^{p} e^{-\gamma|w|^{2}} d A(w) \leq C .
\end{aligned}
$$

The result then follows from an application of Lemma 3.1.

We now have proven that $B M O_{r}^{p}$ is independent of $r$; in what follows, we will write $B M O^{p}=B M O_{1}^{p}$.

TheOREM 1. Let $p \geq 1$. Then the following are equivalent:

(1) $f \in B M O^{p}$;

(2) $f \in B O+B A^{p}$;

$$
\sup _{z \in \mathbb{C}} \int_{\mathbb{C}}\left|f(z-w)-B_{\gamma} f(z)\right|^{p} e^{-\gamma|w|^{2}} d A(w)<\infty,
$$

for some $\gamma>0$; 
(3')

$$
\sup _{z \in \mathbb{C}} \int_{\mathbb{C}}\left|f(z-w)-B_{\gamma} f(z)\right|^{p} e^{-\gamma|w|^{2}} d A(w)<\infty
$$

for all $\gamma>0$;

(4) There is a constant $C$ and $\gamma>0$ such that for every $z \in \mathbb{C}$, there is a constant $\lambda_{z}$ such that

$$
\int_{\mathbb{C}}\left|f(z-w)-\lambda_{z}\right|^{p} e^{-\gamma|w|^{2}} d A(w) \leq C ;
$$

(4') For every $\gamma>0$ there is a constant $C$ such that for every $z \in \mathbb{C}$, there is a constant $\lambda_{z}$ such that

$$
\int_{\mathbb{C}}\left|f(z-w)-\lambda_{z}\right|^{p} e^{-\gamma|w|^{2}} d A(w) \leq C .
$$

Proof. (1) $\Rightarrow$ (2) follows from Lemma 3.10. $(2) \Rightarrow\left(3^{\prime}\right)$ follows from the proof of Lemma 3.11. Obviously $\left(3^{\prime}\right) \Rightarrow(3)$ and $\left(4^{\prime}\right) \Rightarrow(4)$. The proofs of $(3) \Leftrightarrow(4)$ and $\left(3^{\prime}\right) \Leftrightarrow\left(4^{\prime}\right)$ are similar to the proof of Lemma 3.1. $(3) \Rightarrow(1)$ follows from Lemma 3.12 .

Lemma 3.13. If $f \in B M O^{p}$, then $B_{\gamma} f \in B O$, and $f-B_{\gamma} f \in B A^{p}$ for every $\gamma>0$.

Proof. Fix $\gamma>0$. We have

$$
\begin{aligned}
\left|B_{\gamma} f(z)-\widehat{f}_{r}(z)\right| & =\left|B_{\gamma} f(z)-\frac{1}{\pi r^{2}} \int_{D(z, r)} f(w) d A(w)\right| \\
& =\left|\frac{1}{\pi r^{2}} \int_{D(z, r)} B_{\gamma} f(z) d A(w)-\frac{1}{\pi r^{2}} \int_{D(z, r)} f(w) d A(w)\right| \\
& \leq \frac{1}{\pi r^{2}} \int_{D(z, r)}\left|f(w)-B_{\gamma} f(z)\right| d A(w) \\
& \leq C \int_{\mathbb{C}}\left|f(z-w)-B_{\gamma} f(z)\right| e^{-\gamma|w|^{2}} d A(w) .
\end{aligned}
$$

Here the last inequality follows from the proof of Lemma 3.12. Since $f \in B M O^{p}$, the last integral is finite. Thus $B f-\widehat{f}_{r}$ is a bounded continuous function and so lies in $B O \cap B A^{p}$. By Lemma 3.6, $\widehat{f}_{r} \in B O$, so $B_{\gamma} f=B_{\gamma} f-\widehat{f}_{r}+\widehat{f}_{r} \in B O+B O=B O$. By Lemma $3.9, f-\widehat{f}_{r} \in B A^{p}$, so $f-B_{\gamma} f=f-\widehat{f}_{r}+\widehat{f}_{r}-B_{\gamma} f \in B A^{p}+B A^{p}=B A^{p}$.

\section{Bounded Hankel operators}

We begin with a short discussion of harmonic conjugates. If $f=u+i v$ is entire, then both $u$ and $v$ are harmonic. Conversely, given a harmonic $u: \mathbb{C} \rightarrow \mathbb{R}$, there exists a unique harmonic $v: \mathbb{C} \rightarrow \mathbb{R}$ such that $f=u+i v$ is entire and $v(0)=0$. 
Lemma 4.1. Let $u: \mathbb{C} \rightarrow \mathbb{R}$ be harmonic. If $u \in L_{\gamma}^{p}$ for $p \in(1, \infty)$ and $\gamma>0$, then $v \in L_{\gamma}^{p}$.

Proof. Looking at the proof of Theorem 4.1 of [6], one obtains for $r<1$ a $C>0$ such that

$$
\int_{0}^{2 \pi}\left|v\left(r e^{i \theta}\right)\right|^{p} d \theta \leq C \int_{0}^{2 \pi}\left|u\left(r e^{i \theta}\right)\right|^{p} d \theta .
$$

But if $r>1$, consider the dilations $u_{R}(z)=u(R z)$ and $v_{R}(z)=v(R z)$ for large enough $R$. Of course, both $u$ and $u_{R}$ always belong to the hardy space $h^{p}$ of the unit circle. Now,

$$
\int_{0}^{2 \pi}\left|v\left(r e^{i \theta}\right)\right|^{p} d \theta=\int_{0}^{2 \pi}\left|v_{R}\left(s e^{i \theta}\right)\right|^{p} d \theta \leq C \int_{0}^{2 \pi}\left|u_{R}\left(s e^{i \theta}\right)\right|^{p} d \theta=C \int_{0}^{2 \pi}\left|u\left(r e^{i \theta}\right)\right|^{p} d \theta,
$$

where $R$ is chosen so that $s:=r / R<1$. Now, inevitably

$$
\int_{0}^{2 \pi}\left|v\left(r e^{i \theta}\right)\right|^{p} r e^{-(p / 2) r^{2}} d \theta \leq C \int_{0}^{2 \pi}\left|u\left(r e^{i \theta}\right)\right|^{p} r e^{-(p / 2) r^{2}} d \theta .
$$

The rest follows from evaluating the norms in polar coordinates.

Corollary 4.2. Let $p \in(1, \infty)$ and $\gamma>0$. Suppose $f=u+i v$ is entire and that $u \in L_{\gamma}^{p}$. Then $f \in F_{\gamma}^{p}$. Moreover, there exists $C>0$ such that $\|f-f(0)\|_{p, \gamma} \leq$ $\|u\|_{p, \gamma}$.

In what follows, if the possible values of $p$ are not indicated, we assume that $p \in(1, \infty)$.

Recall that the Bergman projection $P$ is given by

$$
P g(z)=\int_{\mathbb{C}} g(w) e^{\gamma z \bar{w}} e^{-|w|^{2}} d A(w) .
$$

If $f \in \tau^{p}$, then the Hankel operator with symbol $f$ is given for $g \in F_{\gamma}^{p}$ by

$$
H_{f} g(z)=(I-P)(f g)(z) .
$$

Note that we can also write

$$
H_{f} g(z)=\int_{\mathbb{C}}(f(z)-f(w)) g(w) e^{\gamma z \bar{w}} e^{-\gamma|w|^{2}} d A(w) .
$$

Lemma 4.3. If $f \in B A^{p}$, then $H_{f}$ is bounded on $F_{\gamma}^{p}$.

Proof. By Lemma 3.8, $M_{f}$ is bounded $F_{\gamma}^{p} \rightarrow L_{\gamma}^{p}$. Since $P$ is bounded, we obtain the desired result.

Lemma 4.4. If $f \in B O$, then $H_{f}$ is bounded on $F_{\gamma}^{p}$ for every $p \in[1, \infty]$. 
Proof. By Lemma 3.4

$$
\left|H_{f}(g)(z)\right| \leq C \int_{\mathbb{C}}(|z-w|+1)\left|e^{\gamma z \bar{w}} g(w)\right| e^{-\gamma|w|^{2}} d A(w) .
$$

There are $C>0$ and $\epsilon>0$ such that

$$
\left|e^{\gamma z \bar{w}}\right| \leq C e^{(\gamma / 2)|z|^{2}+(\gamma / 2)|w|^{2}-\epsilon|z-w|} .
$$

Therefore, we arrive at

$$
\begin{aligned}
& \left|H_{f}(g)(z)\right|^{p} e^{-(p \gamma / 2)|z|^{2}} \\
\leq & C e^{-((p-1) \gamma / 2)|z|^{2}}\left|\int_{\mathbb{C}}(|z-w|+1)\right| g(w)\left|e^{-(\gamma / 2)|w|^{2}} e^{-\epsilon|z-w|} d A(w)\right|^{p} \\
\leq & C e^{-((p-1) \gamma / 2)|z|^{2}}\left\{\int_{\mathbb{C}}|g(w)|^{p} e^{-(\gamma p / 2)|w|^{2}} d A(w)\right\}\left\{\int_{\mathbb{C}}|z-w|^{q} e^{-q \epsilon|z-w|} d A(w)\right\}^{p / q} \\
\leq & C e^{-((p-1) \gamma / 2)|z|^{2}}\|g\|_{p, \gamma}^{p} .
\end{aligned}
$$

If $1<p<\infty$ and $1 / p+1 / q=1$, we get the desired result by integrating with respect to $z$. follows.

If $p=1$, we use the above reasoning together with Fubini and proceed as

$$
\begin{aligned}
& \int_{\mathbb{C}}\left|H_{f}(g)(z)\right|^{p} e^{-(\gamma / 2)|z|^{2}} d A(z) \\
\leq & C \int_{\mathbb{C}}|g(w)| e^{-(\gamma / 2)|w|^{2}} d A(w) \int_{\mathbb{C}}(|z-w|+1) e^{-\epsilon|z-w|} d A(z) \\
\leq & C\|g\|_{1, \gamma} .
\end{aligned}
$$

By similar arguments, one can also show that

$$
\left|H_{f}(g)(z)\right| e^{-(\gamma / 2)|z|^{2}} \leq \int_{\mathbb{C}}(|z-w|+1) e^{-\epsilon|z-w|} d A(z)\|g\|_{\infty, \gamma} \leq C\|g\|_{\infty, \gamma} .
$$

The result is now proven for all $p \in[1, \infty]$.

Theorem 2. Let $f \in \tau^{p}$. Then $f \in B M O^{p}$ if and only if the operators $H_{f}$ and $H_{\bar{f}}$ are both bounded $F_{\gamma}^{p} \rightarrow L_{\gamma}^{p}$.

Proof. If $f \in B M O^{p}$, then so is $\bar{f}$ and it follows from the previous two lemmas that $H_{f}$ and $H_{\bar{f}}$ are bounded.

Suppose now that $H_{f}$ and $H_{\bar{f}}$ are both bounded. Without loss of generality, we may then assume that $f$ is real-valued. Recall that $k_{z}(w)=e^{\gamma \bar{z} w-(\gamma / 2)|z|^{2}}$ are unit vectors in $F_{\gamma}^{p}$ and so we have $C>0$ such that

$$
\left\|f k_{z}-P\left(f k_{z}\right)\right\|_{p, \gamma}=\left\|H_{f}\left(k_{z}\right)\right\|_{p, \gamma} \leq C
$$


Note that $k_{z}(z-w)=1 / k_{z}(w)$ and

$$
e^{-\gamma(p / 2)|z-w|^{2}}=e^{-\gamma(p / 2)|z|^{2}-\gamma(p / 2)|w|^{2}+\gamma(p / 2) z \bar{w}+\gamma(p / 2) \bar{z} w} .
$$

Thus, by a change of variables $w \mapsto z-w$, one obtains

$$
\begin{aligned}
C^{p} & \geq\left\|f k_{z}-P\left(f k_{z}\right)\right\|_{p, \gamma}^{p} \\
& =\int_{\mathbb{C}}\left|f(z-w)-e^{-(\gamma / 2)|z|^{2}} P\left(f k_{z}\right)(z-w)\right|^{p} e^{-(p \gamma / 2)|w|^{2}} d A(w)
\end{aligned}
$$

Setting $g_{z}(w)=e^{-(\gamma / 2)|z|^{2}} P\left(f k_{z}\right)(z-w)$, one obtains

$$
\sup _{z \in \mathbb{C}} \int_{\mathbb{C}}\left|f(z-w)-g_{z}(w)\right|^{p} e^{-(p \gamma / 2)|w|^{2}} d A(w) \leq C^{p} .
$$

Since $f$ is real-valued, then the imaginary part of $g_{z}$ must belong $L_{\gamma}^{p}$ and so

$$
\left\|g_{z}-g_{z}(0)\right\|_{p, \gamma} \leq M
$$

for every $z \in \mathbb{C}$ and some $M>0$. Applying triangle inequality and the main theorem of the previous section with $\lambda_{z}=g_{z}(0)$, one sees that $f \in B M O^{p}$.

\section{VMO and compact Hankel operators}

In this section we study VMO and compactness of Hankel operators. The results and their proofs are completely analogous to the results of the previous two sections. A great deal of details is therefore omitted and left for the reader to verify.

Define $V M O_{r}^{p}$ to be the set of $L_{l o c}^{p}$ integrable functions $f$ such that

$$
\lim _{z \rightarrow \infty} \frac{1}{\pi r^{2}} \int_{D(z, r)}\left|f(w)-\widehat{f}_{r}(z)\right|^{p} d A(w)=0 .
$$

Let $V O_{r} \subset B O_{r}$ be the set of continuous functions in $\mathbb{C}$ such that

$$
\lim _{z \rightarrow \infty} \omega_{r}(f)=0 .
$$

Let $V A_{r}^{p}$ be the set of functions $f$ on $\mathbb{C}$ such that $\lim _{z \rightarrow \infty} \widehat{f}_{r}(z)=0$. The space $V A_{r}^{p}$ is related to the space of vanishing Carleson measures on Fock spaces, see [7], [10].

Similarly to the section 3, it can be shown that $V O_{r}$ and $V A_{r}^{p}$ are independent of $r$ and we will write $V O$ and $V A^{p}$, respectively. The following results are also analogous to the $B M O$-setting.

Lemma 5.1. Let $f \in V M O^{p}$. Then

(1) $B_{\gamma} f \in B O$ for every $\gamma>0$;

(2) $\widehat{f}_{r} \in B O$ for every $r>0$;

(3) $f-B_{\gamma} f \in B A^{p}$ for every $\gamma>0$;

(4) $f-\widehat{f}_{r} \in B A^{p}$ for every $r>0$. 
TheOREM 3. Let $p \geq 1$. Then the following are equivalent:

(1) $f \in V M O^{p}$

(2) $f \in V O+V A^{p}$;

(3)

$$
\lim _{z \rightarrow \infty} \int_{\mathbb{C}}\left|f(z-w)-B_{\gamma} f(z)\right|^{p} e^{-\gamma|w|^{2}} d A(w)=0,
$$

for some $\gamma>0$;

(3')

$$
\lim _{z \rightarrow \infty} \int_{\mathbb{C}}\left|f(z-w)-B_{\gamma} f(z)\right|^{p} e^{-\gamma|w|^{2}} d A(w)=0
$$

for all $\gamma>0$; that

(4) There is a $\gamma>0$ such that for every $z \in \mathbb{C}$, there is a constant $\lambda_{z}$ such

$$
\lim _{z \rightarrow \infty} \int_{\mathbb{C}}\left|f(z-w)-\lambda_{z}\right|^{p} e^{-\gamma|w|^{2}} d A(w)=0
$$

(4') For every $\gamma>0$ and every $z \in \mathbb{C}$, there is a constant $\lambda_{z}$ such that

$$
\lim _{z \rightarrow \infty} \int_{\mathbb{C}}\left|f(z-w)-\lambda_{z}\right|^{p} e^{-\gamma|w|^{2}} d A(w)=0 .
$$

Theorem 4. Let $f \in \tau^{p}$. Then the operators $H_{f}$ and $H_{\bar{f}}$ are compact if and only if $f \in V M O^{p}$.

Proof. Suppose first that $f \in V A^{p}$. But then $|f|^{p} d A$ is vanishing Carleson, so the multiplication operators $M_{f}$ and $M_{\bar{f}}$ are compact $F_{\gamma}^{p} \rightarrow L_{\gamma}^{p}$. From the boundedness of the projection $P$, it follows that $H_{f}$ and $H_{\bar{f}}$ are both compact.

If $f \in V O$, we refer to Lemma 5.1 of [2]. It follows that both $H_{f}$ and $H_{\bar{f}}$ can be approximated in norm by Hankel operators with symbols having a compact support. Therefore, both operators are compact. In conclusion, we have shown that if $f \in V M O^{p}$, then $H_{f}$ and $H_{\bar{f}}$ are compact.

As for the other direction. Note that $k_{z} \rightarrow 0$ weakly, as $z \rightarrow \infty$. But now

$$
\left\|H_{f} k_{z}\right\|_{p, \gamma} \rightarrow 0 \text { and }\left\|H_{\bar{f}} k_{z}\right\|_{p, \gamma} \rightarrow 0,
$$

as $z \rightarrow \infty$. By reasoning similar to that in Theorem 2 , it follows that

$$
\int_{\mathbb{C}}\left|f(z-w)-g_{z}(0)\right|^{p} e^{-(\gamma p / 2)|w|^{2}} d A(w) \rightarrow 0,
$$

as $z \rightarrow \infty$, so $f \in V M O^{p}$.

\section{References}

[1] Bauer, W., Hilbert-Schmidt Hankel operators on the Segal-Bargmann space. Proc. Amer. Math. Soc. 132 (2004), no. 10, 2989-2996 (electronic).

[2] Bauer, W., Mean Oscillation and Hankel Operators on the Segal-Bargmann Space. Integr. equ. oper. theory 52 (2005), 1-15. 
[3] Berndtsson, B.; Ortega Cerdà, J., On interpolation and sampling in Hilbert spaces of analytic functions. J. Reine Angew. Math. 464 (1995), 109-128.

[4] Bommier-Hato, H.; Youssfi, E., Hankel operators on weighted Fock spaces. Integral Equations Operator Theory 59 (2007), no. 1, 1-17.

[5] Dostanic, M.; Zhu, K., Integral operators induced by the Fock kernel. Integral Equations and Operator Theory 60 (2008), 217-236.

[6] Duren, P., Theory of $H^{p}$ spaces. Academic Press, New York, 1970.

[7] Isralowitz, J.; Zhu, K.; Toeplitz operators on the Fock space. Integral Equations and Operator Theory 66 (2010), no. 4, 593-611.

[8] Janson, S.; Peetre, J.; Rochberg, R., Hankel forms and the Fock space. Rev. Mat. Iberoamericana 3 (1987), no. 1, 61-138.

[9] Peller, V., Hankel operators and their applications. Springer-Verlag, New York, 2003.

[10] Schuster, A.; Varolin, D., Toeplitz Operators and Carleson Measures on Generalized Bargmann-Fock Spaces. Integral Equations and Operator Theory, Volume 72, Number 3 (2012), 363-392.

[11] Taskinen, J.; Virtanen, J. A., Weighted BMO and Toeplitz operators on the Bergman space $A^{1}$. (to appear in Journal of Operator Theory).

[12] Zhu, K., BMO and Hankel operators on Bergman spaces. Pacific J. Math. 155 (1992), 377-395.

A. Perälä

Department of Mathematics

University of Helsinki

Helsinki 00014

Finland

e-mail: antti.i.perala@helsinki.fi

A. Schuster

${ }^{\star}$ Department of Mathematics

San Francisco State University

San Francisco, CA 94132

USA

${ }^{\star}$ Department of Mathematics and Actuarial Science

The American University in Cairo

AUC Avenue PO Box 74

New Cairo Egypt 11835

e-mail: schuster@sfsu.edu

J. A. Virtanen

Department of Mathematics

University of Bristol

Bristol, England BS8 1TW

UK

e-mail: virtanen@cims.nyu.edu 